\title{
Disregulation of E-cadherin in transgenic mouse models of liver cancer
}

\author{
Diego F Calvisi*, Sara Ladu*, Elizabeth A Conner, Valentina M Factor and \\ Snorri S Thorgeirsson \\ Laboratory of Experimental Carcinogenesis, National Cancer Institute, Bethesda, MD, USA
}

\begin{abstract}
E-cadherin is a cell-cell adhesion molecule that plays a pivotal role in the development and maintenance of cell polarity. Disruption of E-cadherin-mediated adhesion represents a key step toward the invasive phenotype in a variety of solid tumors, including hepatocellular carcinoma (HCC). Here, we investigate whether deregulation of E-cadherin occurs along the multistep process of hepatocarcinogenesis in transgenic mouse models, including C-Myc, E2F1, c-Myc/TGF- $\alpha$ and c-Myc/E2F1 mice. Liver tumors from the transgenic mouse lines could be divided into two categories based on E-cadherin levels. Of 28, 20 (71.4\%) c-Myc HCCs showed marked reduction of E-cadherin expression when compared with wild-type livers. In contrast, all of c-Myc/TGF- $\alpha$ and the majority of E2F1 and c-myc/E2F1 preneoplastic and neoplastic lesions exhibited overexpression of E-cadherin. Downregulation of E-cadherin was associated with promoter hypermethylation in seven of 20 c-Myc HCCs (35\%), while no loss of heterozygosity at the E-cadherin locus was detected. Nuclear accumulation of $\beta$-catenin did not correlate with E-cadherin downregulation. Furthermore, c-Myc HCCs with reduced E-cadherin displayed upregulation of hypoxia-inducible factor- $1 \alpha$ and vascular endothelial growth factor proteins. Importantly, loss of E-cadherin was associated with increased cell proliferation and higher microvessel density in c-Myc tumors. Taken together, these data suggest that loss of E-cadherin might favor tumor progression in relatively more benign HCC from c-Myc transgenic mice by stimulating neoplastic proliferation and angiogenesis under hypoxic conditions.

Laboratory Investigation (2004) 84, 1137-1147, advance online publication, 28 June 2004; doi:10.1038/labinvest.3700147
\end{abstract}

Keywords: angiogenesis; $\beta$-catenin; E-cadherin; HCC; hypoxia; transgenic mice

Hepatocellular carcinoma (HCC), one of the most common solid tumors worldwide, is characterized by poor prognosis and reduced survival. ${ }^{1,2}$ The severity of the clinical outcome depends upon the potential for invasiveness of the primary HCC, leading to a high rate of intrahepatic recurrence after treatment. At the clinical level, the invasive potential of HCC is determined by its ability to invade the Glisson's capsule or portal vein., However, the molecular mechanisms through which HCC acquires the invasive potential remain poorly understood.

Alterations affecting cell adhesion molecules are considered to play a critical role in the invasive process. Among these molecules, E-cadherin, a member of the cadherin family, is involved in

Correspondence: Dr SS Thorgeirsson, MD, PhD, Laboratory of Experimental Carcinogenesis, National Cancer Institute, 37 Convent Drive MSC 4262, Bethesda, MD 20892-4262, USA.

E-mail: snorri thorgeirsson@nih.gov

*These two authors contributed equally to the work

Received 22 March 2004; revised and accepted 4 May 2004; published online 28 June 2004 homotypic, calcium-dependent cell-cell adhesion in epithelial tissues. ${ }^{5}$ Physiologically, E-cadherin regulates a variety of morphogenetic events, including cell migration, separation and formation of boundaries between cell layers and differentiation of each cell layer into functionally distinct structures. The E-cadherin intracellular domain interacts with $\beta$-catenin and plakoglobin, which in turn bind to $\alpha$-catenin. ${ }^{5}$ The cadherin-catenin complex is connected through $\alpha$-catenin to the actin filaments, forming a stable adherent junction. Loss or reduced expression of E-cadherin results in dedifferentiation, invasiveness, lymph node or distant metastasis in a variety of human neoplasms, including HCC. ${ }^{6-10}$ Accordingly, in vitro studies have shown that Ecadherin overexpression inhibits cellular growth, transformation and invasiveness. ${ }^{11,12}$ Furthermore, forced expression of E-cadherin blocked the transition from adenoma to carcinoma in a transgenic model of pancreatic $\beta$-cell carcinogenesis. ${ }^{13}$ Downregulation of E-cadherin is achieved by genetic mutations in combination with loss of heterozygosity $(\mathrm{LOH})$ at the E-cadherin locus in carcinomas of the stomach, ovary, endometrium and breast. ${ }^{14}$ In 
human HCC, inactivation of E-cadherin is often associated with $\mathrm{LOH}$ at the E-cadherin locus or epigenetic mechanisms. The latter include E-cadherin promoter hypermethylation or binding of transcriptional repressors such as Snail or Slug. ${ }^{15-19}$ This body of evidence suggests that E-cadherin acts as a canonical tumor suppressor gene. However, recent reports indicate that the role of E-cadherin might be more complex than previously believed. Indeed, overexpression of E-cadherin has also been linked with the invasive potential of tumor cells, ${ }^{19-22}$ implying that enforced intercellular adhesion mediated by E-cadherin might favor the outgrowth of neoplastic clones with invasive properties. ${ }^{19-22}$

We have previously generated a number of transgenic mouse models prone to liver cancer, including c-Myc, E2F1, c-Myc/TGF- $\alpha$ and c-Myc/ E2F1. ${ }^{23-25}$ These transgenic mice developed HCC with different morphological and biological features. In particular, coexpression of c-Myc with TGF- $\alpha$ or E2F1 resulted in a shorten tumor latency, higher incidence as well as in a more aggressive phenotype when compared with single transgenic lines. ${ }^{23-25}$ In order to elucidate the role of E-cadherin in the sequential steps of liver carcinogenesis, we have analyzed the expression patterns of E-cadherin in a collection of preneoplastic and neoplastic liver lesions from c-Myc, E2F1, c-Myc/TGF- $\alpha$ and c-Myc/ E2F1 transgenic mice. In particular, we have investigated the relevance of genetic, epigenetic and transcriptional mechanisms on E-cadherin protein expression levels. Our data indicate that loss of E-cadherin contributes to HCC progression in c-Myc transgenic mice by promoting cell proliferation and angiogenesis, presumably through the upregulation of HIF- $1 \alpha$ and VEGF proteins.

\section{Materials and methods}

\section{Transgenic Mice}

Generation of the Alb/c-Myc (c-Myc), Alb/E2F1 single-transgenic mice and Alb/c-myc/MT/TGF- $\alpha$ (c-myc/TGF- $\alpha$ ) double-transgenic mice has been previously described. ${ }^{23,24}$ Double transgenic Alb/ C-myc/Alb/E2F1 (c-Myc/E2F1) mice were generated by crossing homozygous E2F1 (line 8) with homozygous c-Myc (line 166.8) mice. ${ }^{25}$ Animal housing and care were in accordance with National Institutes of Health guidelines.

\section{Tissue Specimens, Histopathological Analysis and DNA Extraction}

Normal, preneoplastic and neoplastic liver tissues were obtained from male mice at different ages (3-20 months). They were dissected and divided into two parts: half was stored at $-80^{\circ} \mathrm{C}$ and the other half was fixed in $10 \%$ formalin and embedded in paraffin blocks. Sections were stained with hematoxylin and eosin (H\&E). Histopathological diagnoses were based upon criteria described in Frith et $a{ }^{26}$ and high molecular weight DNA was isolated from frozen mouse liver and tumor tissues as reported. ${ }^{27}$

\section{Immunohistochemistry}

Immunohistochemical staining was performed on $10 \%$ formalin-fixed, paraffin-embedded sections. Deparaffinized sections were incubated in $3 \%$ $\mathrm{H}_{2} \mathrm{O}_{2}$ dissolved in methanol for $20 \mathrm{~min}$ and microwaved in Antigen Unmasking Solution (Dako Corporation, Carpinteria, CA, USA) for $12 \mathrm{~min}$. The mouse monoclonal anti- $\beta$-catenin, anti-E-cadherin (Transduction Laboratories, Lexington, KY, USA; dilution 1:100), anti-PCNA (Santa Cruz Biotechnology, Santa Cruz, CA, USA; 1:1000), anti-CD34 (Vector Laboratories, Burlingame, CA, USA; 1:40) and rabbit polyclonal anti-phosphohistone H3 (Upstate USA, Lake Placid, NY, USA; 1:250) antibodies were applied. Immunoreactivity was visualized with the Vectastain Elite ABC kit (Vector Laboratories) and 3,3' DAB (Dako Corporation) as the chromogen. Slides were counterstained with Gill's hematoxylin. For E-cadherin staining, immunolabeling of the interlobular biliary epithelial cells was used as internal positive control. Intensity of Ecadherin immunoreactivity in preneoplastic and tumor samples was graded by comparison with staining obtained in eight wild-type livers. The scores were determined as follows: loss, when the lesion displayed marked reduction or loss of immunostaining compared to the normal livers; unchanged, when the immunoreactivity was similar between wild-type and transgenic lesions; overexpression, when intensity was higher in the transgenic lesion compared to the wild-type livers.

\section{Western Blot Analysis}

Hepatic tissue samples were homogenized and sonicated in lysis buffer as previously described. ${ }^{28}$ Protein concentration was determined with the BioRad Protein Assay Kit (Bio-Rad, Hercules, CA, USA), using bovine serum albumin as standard. Aliquots of $50 \mu \mathrm{g}$ were denatured by boiling in TrisGlycine SDS Sample Buffer (Novex, San Diego, CA, USA), separated by SDS-PAGE and transferred onto nitrocellulose membranes by electroblotting. The following antibodies were applied: mouse monoclonal anti-E-cadherin (Transduction Laboratories; 1:1000), anti-HIF-1 $\alpha$ (Novus Biologicals, Littleton, CO, USA; 1:1000), anti-VEGF (Santa Cruz Biotechnology, Santa Cruz, CA, USA; 1:200), goat polyclonal anti-Snail (Santa Cruz Biotechnology), rabbit polyclonal anti-Slug and anti-Wilms' tumor-1 (WT1) (Santa Cruz Biotechnology; 1:200). Each primary antibody was followed by incubation with horse 
radish peroxidase (HRP)-secondary antibody diluted 1:5000 for $1 \mathrm{~h}$ and revealed with the Super Signal West Pico (Pierce Biotechnology Inc., Rockford, IL, USA). Equal loading was assessed by probing the same membranes with the mouse anti-actin antibody (Chemicon International, Temecula, CA, USA; 1:2000).

\section{Microsatellite Analysis of the E-cadherin Locus}

Eight 4-month-old wild-type mice (4 B6CBA and 4 [B6CBA $\times$ CD1] F1) were used as controls and 88 HCCs (28 tumors from c-Myc, 20 tumors each from E2F1, c-Myc/TGF- $\alpha$, and c-Myc/E2F1, respectively) were analyzed for microsatellite alterations at the E-cadherin locus through D8Mit151, D8Mit313, D8Mit12, and D8Mit138 primers, which were purchased from Research Genetics (Huntsville, AL, USA). The microsatellite primer sequences and product sizes are shown in Table 1. The PCR was carried out in a reaction volume of $10 \mu \mathrm{l}$ consisting of $0.4 \mu \mathrm{M}$ each primer, $200 \mu \mathrm{M}$ each dNTP, $10 \times$ PCR buffer, $0.4 \mathrm{U}$ of FastStart Taq DNA Polymerase (Roche Molecular Biochemicals, Indianapolis, IN, USA) and $20 \mathrm{ng}$ of template DNA, with an initial activation step at $95^{\circ} \mathrm{C}$ for $6 \mathrm{~min}$, followed by 35 cycles $\left(95^{\circ} \mathrm{C}\right.$ for $30 \mathrm{~s}, 55^{\circ} \mathrm{C}$ for $45 \mathrm{~s}, 72^{\circ} \mathrm{C}$ for $\left.1 \mathrm{~s}\right)$ and a final extension at $72^{\circ} \mathrm{C}$ for $10 \mathrm{~min}$. After denaturation at $99^{\circ} \mathrm{C}$ in TBE-Urea Sample Buffer (Novex), $12 \mu \mathrm{l}$ of each mixture were electrophoresed at $170 \mathrm{~V}$ for $50 \mathrm{~min}$ and visualized by SYBR Green I Nucleic Acid Gel Stain (Novex) according to the manufacturer's protocol. LOH was recorded when a $50 \%$ or greater reduction in electrophoretic band intensity was detected. Microsatellite instability (MSI) was identified by the presence of band shifts or novel bands in the PCR product.

\section{Methylation-Specific PCR Analysis of the E-cadherin Promoter}

Genomic DNA from wild-type and transgenic mice was modified by using the CPGenome DNA Modification Kit (Serologicals Corporation, Norcross, GA, USA). Primer pairs were designed to detect the unmethylated and the methylated status of the
E-cadherin promoter (Table 1). Primers amplify the entire GC-rich region of the E-cadherin promoter, which has been previously characterized..$^{29,30} \mathrm{~A}$ total of $30 \mathrm{ng}$ of DNA was amplified in a reaction volume of $10 \mu \mathrm{l}$ consisting of $0.4 \mu \mathrm{M}$ each primer, $200 \mu \mathrm{M}$ each dNTP, $10 \times$ PCR buffer, $5 \times$ GC-RICH solution, and $0.4 \mathrm{U}$ of FastStart Taq DNA Polymerase (Roche Molecular Biochemicals), with an initial activation step at $95^{\circ} \mathrm{C}$ for $6 \mathrm{~min}$, followed by 35 cycles $\left(95^{\circ} \mathrm{C}\right.$ for $30 \mathrm{~s}, 60^{\circ} \mathrm{C}$ for $45 \mathrm{~s}, 72^{\circ} \mathrm{C}$ for $1 \mathrm{~s}$ ) and a final extension at $72^{\circ} \mathrm{C}$ for $10 \mathrm{~min}$. E-cadherin promoter methylation was confirmed by direct sequencing of both strands by using the same set of methylationspecific primers.

\section{Quantitation of Mitotic and PCNA Indices, Tumor Size}

Mitotic and PCNA indices were scored on c-Myc HCCs from eight mice per group and at least 2000 hepatocyte nuclei per animal were counted. Indices were determined by counting phosphohistone H3and PCNA-positive cells after counterstaining with hematoxylin, respectively. At least 2000 nuclei were counted per mouse. The indices were represented as a percentage (mean + s.e.) of the total cells counted. Tumor size was measured in $\mathrm{mm}^{2}$ on H\&E-stained sections obtained from the central part of HCCs using the ImageJ software.

\section{Quantitation of Apoptosis}

Apoptotic index was calculated by counting the apoptotic figures per at least 2000 hepatocytes on tumor sections. Eight mice per group were evaluated. Sections were stained with the ApoTag peroxidase in situ apoptosis detection kit (Serologicals Corporation) and expressed as percentage of the total number of counted cells. Indices were represented as a percentage (mean + s.e.) of the total cells counted.

\section{Evaluation of Microvessel Density}

Eight HCC per group were subjected to immunostaining with mouse monoclonal anti-CD34 antibody (Vector Laboratories). The tumors were first

Table 1 Sequences of microsatellite, E-cadherin promoter unmethylated (U)- and methylated (M)-specific primers

\begin{tabular}{|c|c|c|c|}
\hline \multirow[t]{2}{*}{ Primer name } & \multicolumn{2}{|c|}{ Sequence } & \multirow[t]{2}{*}{ Product size $(b p$} \\
\hline & Forward & Reverse & \\
\hline D8Mit151 & CCTGGAAAGGGAAAACATT & TTTCCTCTGACCTCCAATGG & $244-264$ \\
\hline D8Mit313 & CCTGCTTAAATGCCGACACT & GGGACATGGCTGGTATAAATT & 125 \\
\hline D8Mit12 & GATCTCTACATCAAAAGGGA & TTCAGTTTTGTTTCTGAAAC & 119 \\
\hline D8Mit138 & ATCCTTCAGAGGACCGGAGT & CACCATGCCTGACATTTCTG & 192 \\
\hline E-CAD U & СССАACСAATCAACAACACCAАAAACAA & GGTTTTTATGGTGGATTTGGTAGATGTT & 206 \\
\hline E-CAD M & CAACCAATCAACGACGCCGAAAACGA & TTTTTATGGCGGATTCGGTAGACGTC & 202 \\
\hline
\end{tabular}


screened at low power $(\times 40)$ to identify the areas of highest microvessel density (MVD). The four highest MVD areas for each tumor were photographed at high power $(\times 200)$ and the size of each area standardized using the ImageJ software. Any brown-stained endothelial cell or endothelial cell cluster was counted as one microvessel, irrespective of the presence of a vessel lumen. MVD was expressed as the percentage (mean + s.e.) of the total CD-34-stained spots per section area $\left(0.94 \mathrm{~mm}^{2}\right)$.

\section{Statistical Analysis}

Student's $t$-test was applied to evaluate statistical significance. Values of $P<0.05$ were considered to be statistically significant.

\section{Results}

\section{Histopathology and Incidence of HCC in Transgenic Mice}

Since overexpression of TGF- $\alpha$, c-Myc and E2F1 has been frequently found in human and rodent HCC, ${ }^{23-25,31}$ we have generated c-Myc, c-Myc/TGF- $\alpha$, E2F1, and c-Myc/E2F1 transgenic mice to investigate the role played by these genes in the malignant growth of the liver. ${ }^{23-25,31}$ Although both single transgenic lines were prone to liver cancer, c-Myc mice developed tumors more slowly and with lower incidence than E2F1 mice (Table 2). Coexpression of two transgenes, either c-Myc/E2F1 or c-Myc/TGF- $\alpha$, significantly accelerated the course of HCC development. In double transgenic mice, tumors arose faster and were more malignant than in either of single transgenic mice (Table 2; Calvisi et $a{ }^{28}$ ). HCCs arising in c-Myc/TGF- $\alpha$ mice were particular aggressive and most of them died by 12-14 months of age. ${ }^{23}$

\section{E-cadherin Immunohistochemistry and Western Blot Analysis}

In order to determine E-cadherin expression in wildtype and transgenic livers, immunohistochemical analysis was performed. Ductular epithelial cells showed intense E-cadherin immunolabeling in the portal space. Wild-type and dysplastic hepatocytes from c-Myc transgenic mice exhibited a faint Ecadherin immunoreactivity limited to the basal membrane (Figure 1a) (Table 3). In striking contrast, dysplastic hepatocytes from E2F1, c-Myc/TGF- $\alpha$ and c-Myc/E2F1 displayed increased membranous staining (Figure 1b). Accordingly, HCCs from different transgenic lines segregated in two different categories based on E-cadherin expression levels. Loss of E-cadherin immunostaining was detected in the majority of c-Myc (20/28, 71.4\%; Figure 1c, d) and in a subset of c-Myc/E2F1 HCCs $(4 / 20,20 \%)$, whereas it was not observed in E2F1 and c-Myc/TGF- $\alpha$ liver tumors. Nonetheless, all c-Myc/TGF- $\alpha$, and most E2F1 (17/20, 85\%) and c-Myc/E2F1 (16/20, 80\%) malignant lesions showed a remarkable increase of E-cadherin membranous immunoreactivity (Figure 1d). The difference in expression levels of Ecadherin in the four transgenic lines was confirmed by Western blot analysis (Figure 2). In particular, the highest levels of E-cadherin expression were observed in the more malignant HCCs from c-Myc/ TGF- $\alpha$ transgenic mice. These data indicate that downregulation of E-cadherin is a signature of tumor progression in the majority of c-Myc transgenic mice, whereas increased E-cadherin is characteristic of c-Myc/TGF- $\alpha$, E2F1, and c-Myc/E2F1 preneoplastic and neoplastic lesions.

Table 2 Summary of HCC onset, incidence and tumor phenotype in different transgenic mouse models

\begin{tabular}{lccc}
\hline Transgenic line & HCC onset (months) & HCC incidence (age) & Tumor phenotype \\
\hline E2F1 & 9 & 70 (14 months) & Well-differentiated \\
C-Myc/E2F1 & 6 & 100 (9 months) & Moderately differentiated \\
C-Myc/TGF- $\alpha$ & 3 & 100 (8 months) & Moderately/poorly differentiated \\
C-Myc & 10 & 60 (18 months) & Well-differentiated
\end{tabular}

\footnotetext{
Figure 1 Immunohistochemistry of E-cadherin and $\beta$-catenin expression in preneoplastic and neoplastic lesions developed in different transgenic lines. (a) c-Myc eosinophilic focus displaying comparable levels of E-cadherin immunostaining with wild-type liver (inset). (b) c-myc/TGF- $\alpha$ dysplastic lesion showing increased immunoreactivity for E-cadherin. Of note, overexpression of E-cadherin is mainly located in pericentral areas (inset). (c) c-Myc/E2F1 HCC (indicated by arrows) exhibiting loss of E-cadherin immunoreactivity, whereas the surrounding tissue (left part of the figure) retains E-cadherin immunolabeling. (d), E2F1 clear cell carcinoma showing intense immunostaining for E-cadherin. c-Myc solid HCC displaying concomitant loss of E-cadherin (e) and nuclear and/or cytoplasmic accumulation of $\beta$-catenin (f and inset, $\times 200$ ). Note that biliary epithelial cells (indicated by the arrow) retain the staining for E-cadherin. c-Myc HCC with loss of E-cadherin immunoreactivity (g) retaining membranous $\beta$-catenin immunolocalization (h). Original magnifications, $\times 200$ in (a) and (b); $\times 100$ in (c), (d), (g) and (h); $\times 40$ in (e) and (f). Histochemical staining was performed on serial sections in (e) and (f), and (g) and (h).
} 

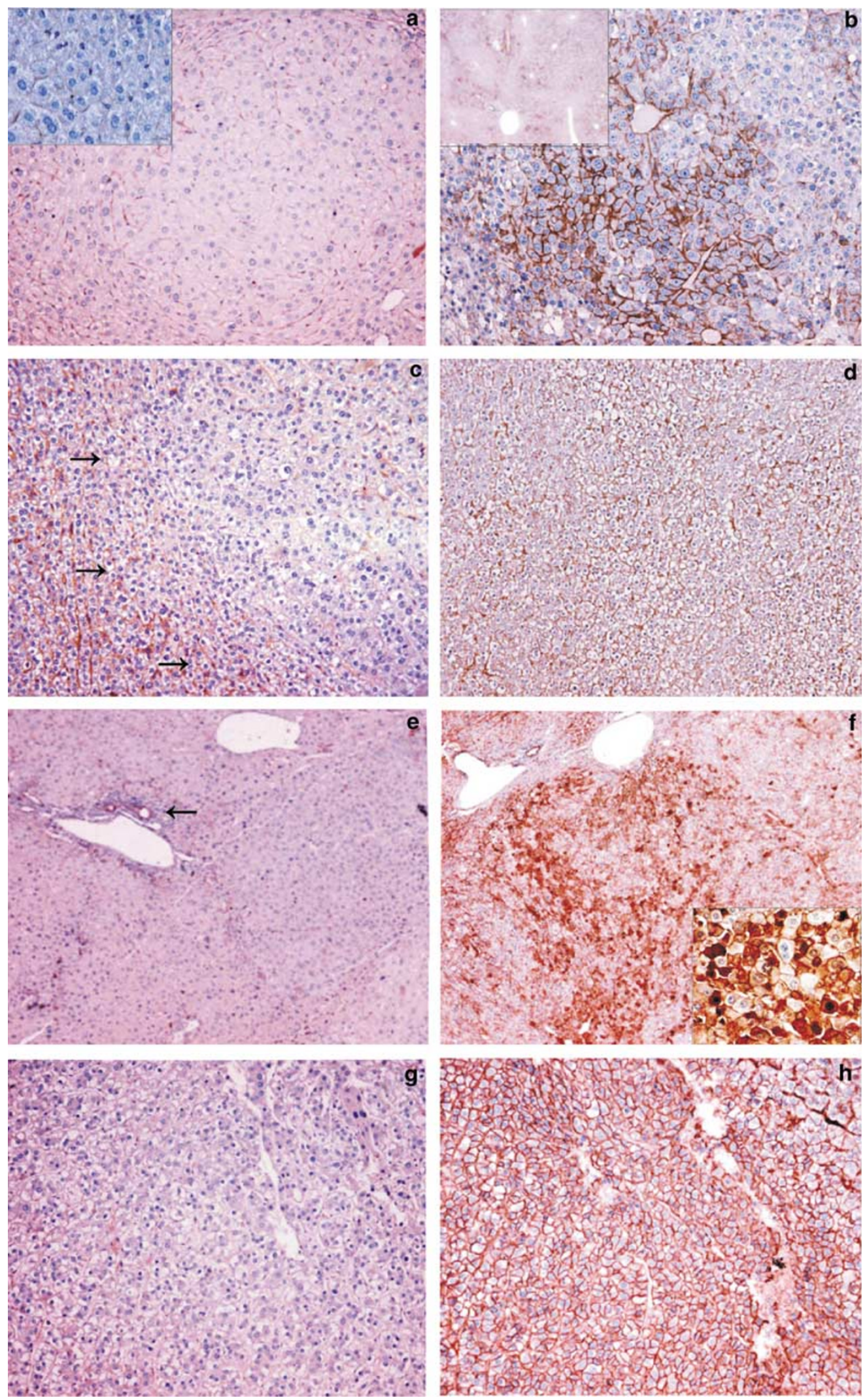
Table 3 Summary of E-cadherin and $\beta$-catenin immunohistochemistry in preneoplastic and neoplastic lesions developed in different transgenic lines

\begin{tabular}{|c|c|c|c|c|c|c|c|c|}
\hline \multirow[t]{2}{*}{ Transgenic line } & \multicolumn{2}{|c|}{$\begin{array}{c}\text { E-cadherin } \\
\text { downregulation }\end{array}$} & \multicolumn{2}{|c|}{$\begin{array}{l}\text { E-cadherin } \\
\text { unchanged }\end{array}$} & \multicolumn{2}{|c|}{$\begin{array}{c}\text { E-cadherin } \\
\text { overexpression }\end{array}$} & \multicolumn{2}{|c|}{$\begin{array}{l}\beta \text {-catenin nuclear } \\
\text { accumulation }\end{array}$} \\
\hline & Dysplasia & $H C C$ & Dysplasia & HCC & Dysplasia & HCC & Dysplasia & HCC \\
\hline E2F1 $(n=20)$ & 0 & 0 & 3 & 3 & 17 & 17 & 0 & 1 \\
\hline c-Myc/E2F1 $(n=20)$ & 0 & 4 & 4 & 0 & 16 & 16 & 14 & 15 \\
\hline c-Myc/TGF- $\alpha(n=20)$ & 0 & 0 & 0 & 0 & 20 & 20 & 0 & 2 \\
\hline c-Myc $(n=28)$ & 0 & 20 & 28 & 8 & 0 & 0 & 9 & 10 \\
\hline
\end{tabular}

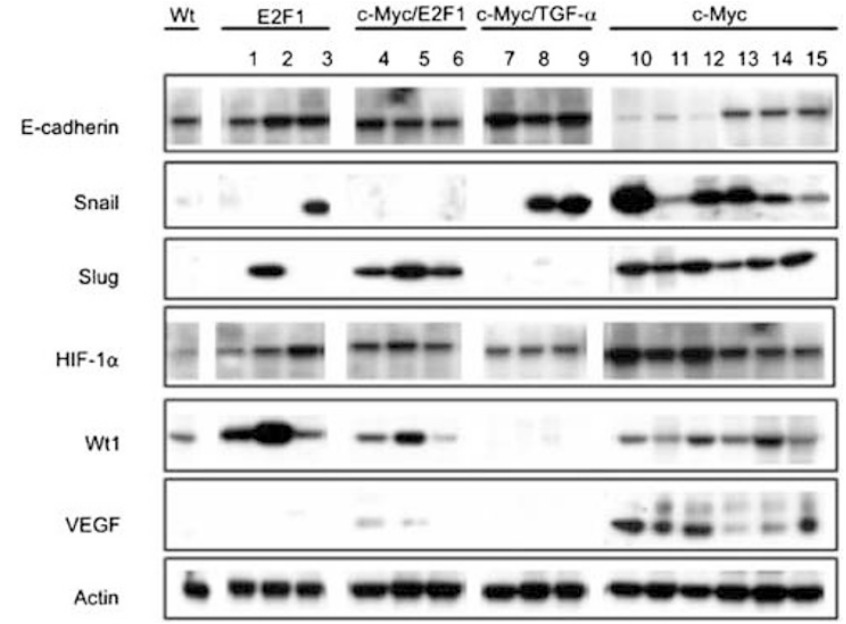

Figure 2 Representative Western blot analysis of E-cadherin and E-cadherin-interacting proteins in wild-type livers (Wt) E2F1 (lanes 1-3), c-Myc/E2F1 (lanes 4-6), c-Myc/TGF- $\alpha$ (lanes 7-9) and c-Myc (lanes 10-15) HCCs.

\section{LOH and Promoter Methylation Status Analysis}

Since reduction or loss of E-cadherin expression is primarily determined by $\mathrm{LOH}$ at the E-cadherin locus or by its promoter hypermethylation in human HCC, ${ }^{16,17,19}$ we have determined the status of the Ecadherin locus and promoter methylation in the same collection of wild-type livers and tumors from transgenic mice by microsatellite analysis and methylation-specific-PCR (MSP-PCR), respectively. Wild-type livers and HCCs, regardless of their origins, showed the absence of $\mathrm{LOH}$ at the $\mathrm{E}$ cadherin locus (Figure 3a,b). E-cadherin promoter was not hypermethylated in wild-type, c-Myc/TGF$\alpha$ and E2F1 livers. No E-cadherin promoter hypermethylation was detected in c-Myc and c-Myc/E2F1 HCCs with normal levels of E-cadherin protein. In striking contrast, seven of $20(35 \%)$ of c-Myc and two of four (50\%) of c-Myc/E2F1 HCCs with downregulation of E-cadherin displayed E-cadherin promoter hypermethylation (Figure 3c). These results suggest that promoter hypermethylation might be responsible for E-cadherin downregulation in a subset of c-Myc and c-Myc/E2F1 HCCs.

\section{Western Blot Analysis of Snail, Slug, HIF-1 $\alpha$, VEGF and WT1}

Recent studies have shown that E-cadherin expression can be suppressed in cancer by specific transcription factors, including Snail, Slug, SIP1 and E12/E47. ${ }^{15,18,19,32-35}$ In particular, Snail downregulates the expression of E-cadherin by induction of hypoxia-inducible factor- $\alpha$ (HIF- $\left.1 \alpha ;{ }^{36}\right)$. On the other hand, WT1 directly transactivates E-cadherin, suggesting that loss of WT1 may also repress the Ecadherin gene. ${ }^{37}$ In order to assess whether similar relationships exist in mouse hepatocarcinogenesis, we have determined the expression levels of Snail, Slug, HIF-1 $\alpha$ and WT1 by Western blot analysis in the same collection of mouse HCCs. The results of our analysis are shown in Figure 2. Snail expression was detected in all (28/28) c-Myc HCCs regardless of E-cadherin protein levels. In addition, 2/20 (10\%) E2F1 and 6/20 (30\%) c-Myc/TGF- $\alpha$ tumors displayed induction of Snail. High levels of Slug were observed in all c-Myc and c-Myc/E2F1 HCCs, whereas it was more rarely upregulated in E2F1 (4/ $20,20 \%$ ) and not induced in c-Myc/TGF- $\alpha$ corresponding liver lesions. HIF- $1 \alpha$ was upregulated in all HCCs from the four transgenic lines. However, the highest levels of HIF- $1 \alpha$ were observed in C-Myc and $\mathrm{c}-\mathrm{Myc} / \mathrm{E} 2 \mathrm{~F} 1 \mathrm{HCCs}$ with reduced levels of Ecadherin, suggesting that E-cadherin loss reinforces HIF- $1 \alpha$ induction. Since hypoxia upregulates the expression of the proangiogenic factor VEGF, ${ }^{38,39}$ we have determined the levels of VEGF protein in the same samples. Strikingly, c-Myc and c-Myc/E2F1 HCCs with low E-cadherin displayed high levels of VEGF, whereas VEGF was not induced in c-Myc/ TGF- $\alpha$, E2F1 and in the majority of c-Myc/E2F1 lesions with overexpression of E-cadherin. c-Myc HCCs with an intact E-cadherin also showed induction of VEGF, but less pronounced. Therefore, similar to HIF-1 $\alpha$, E-cadherin loss further stimulates the induction of VEGF. Furthermore, we have determined WT1 expression levels in all transgenic lines. All c-Myc, E2F1 and c-Myc/E2F1 malignant lesions did not show any significant differences in the levels of WT1 protein when compared with wild type. In striking contrast, WT1 protein was not expressed in c-myc/TGF- $\alpha$ HCCs, in accordance 

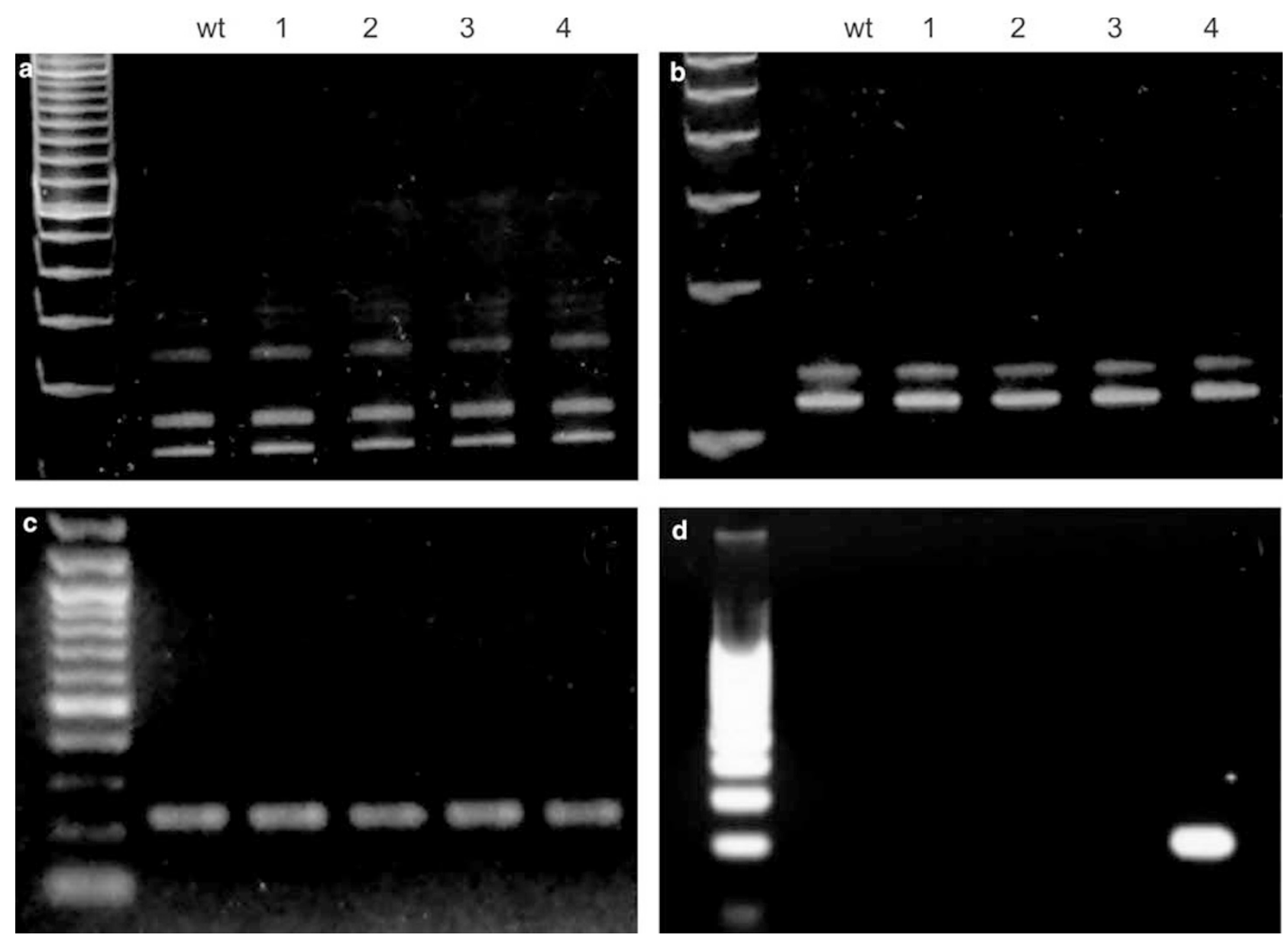

Figure 3 Microsatellite analysis of the E-cadherin locus using the polymorphic markers D8 Mit151 (a) and D8Mit313 (b) in wild-type livers and HCCs from transgenic mice. (c, d) MS-PCR of the same samples using the unmethylated-specific (c) and methylated-specific (d) primers. Wt, wild-type liver; lane 1, E2F1; lane 2, c-Myc/E2F1; lane 3, c-Myc/TGF- $\alpha$; lane 4, c-Myc.

with the frequent LOH at the WT1 locus in the same transgenic mice. ${ }^{40}$ Although E-cadherin is a WT1 direct target gene, ${ }^{37}$ the present findings indicate that E-cadherin expression can be regulated by alternative pathways in the liver, as previously described in Wilms' tumors. ${ }^{41}$

\section{Comparative Immunohistochemical Analysis of E-cadherin and $\beta$-catenin}

A body of evidence indicates that disruption of E-cadherin/catenin binding leads to nuclear translocation of $\beta$-catenin. ${ }^{41-43}$ In order to assess whether E-cadherin downregulation is associated with activation of $\beta$-catenin, we have determined the cellular localization of $\beta$-catenin in the same collection of preneoplastic and neoplastic lesions. As shown in Table 2, $\beta$-catenin activation displayed a different staining pattern when compared with E-cadherin immunohistochemical data. Nuclear accumulation of $\beta$-catenin was extremely frequent in c-Myc/E2F1 preneoplastic $(14 / 20,70 \%)$ and neoplastic lesions $(15 / 20,75 \%)$. c-Myc preneoplastic and neoplastic lesions displayed an intermediate incidence (32.1 and $35.7 \%$, respectively), whereas $\beta$-catenin activation was rare and limited to HCCs in c-Myc/TGF- $\alpha$ and E2F1 (2/20 and 1/20, respectively) transgenic mice. In c-Myc and c-Myc/E2F1 transgenic mice, $\beta$-catenin nuclear accumulation was detectable in preneoplastic focal lesions in accordance with our and others previous findings, ${ }^{28,39,44-46}$ whereas loss or reduction of E-cadherin was present only in fully malignant HCCs (Figure 1c, e). Furthermore, only a fraction $(5 / 20,25 \%)$ of $\mathrm{c}-\mathrm{Myc}$ HCCs with nuclear accumulation of $\beta$-catenin displayed a concomitant E-cadherin downregulation (Figure 1e,f) suggesting that loss of E-cadherin is per se not able to induce nuclear translocation of $\beta$-catenin, as described in human HCC. ${ }^{19}$ Taken together, these results show that $\beta$-catenin activation and suppression of $\mathrm{E}$ cadherin are two distinct and independent mechanisms in c-Myc-induced hepatocarcinogenesis.

\section{Cell Proliferation, Apoptosis and Tumor Size}

A body of evidence suggests that E-cadherin is involved in the control of both cell proliferation and 
apoptosis. ${ }^{11-13,47}$ To better define the functional consequence of E-cadherin downregulation during c-Myc-driven hepatocarcinogenesis, we have determined the mitotic and apoptotic indices in HCCs from c-Myc transgenic mice with reduced or normal levels of E-cadherin. The mitotic index was significantly higher in c-Myc HCCs with E-cadherin downregulation when compared with HCCs with unchanged E-cadherin (1.29 and 0.98, respectively; $P<0.0005$; Figure 4a). The same difference was revealed by the PCNA-labeling index (20.36 and 15.21, respectively; $P<0.0007$; Figure 4b). Furthermore, c-Myc HCCs with downregulation of Ecadherin exhibited a significantly larger tumor size when compared with E-cadherin wild-type HCCs (128.1 and 100.1, respectively; $P=8.95 \mathrm{E}-05$; Figure 4e). In contrast, the apoptotic index was similar among the two groups of HCCs (1.88 and 1.84, respectively; $P=0.75$; Figure 4c). Collectively, these data indicate that loss of E-cadherin is associated with increase in cell proliferation but not with inhibition of apoptosis during c-Myc liver carcinogenesis.

\section{Microvessel Density}

Since downregulation of E-cadherin was associated with increased protein levels of the proangiogenic proteins HIF- $1 \alpha$ and VEGF, we have determined MVD in c-Myc HCCs with low or normal levels of E-cadherin. Strikingly, the MVD index was significantly higher in HCCs with downregulation of E-cadherin when compared with tumors with unchanged E-cadherin (102.98 and 74.08, respectively; $\quad P<0.0003$; Figure $4 \mathrm{~d}$ ). Taken together, these results suggest that reduction of E-cadherin might contribute to angiogenesis in c-Myc HCCs.

\section{Discussion}

Deregulation of E-cadherin expression has been established as an important step during tumorigenesis in a variety of human tumors, including HCC. ${ }^{6-10}$ Here, we show that loss of E-cadherin is a molecular signature of HCC progression in the more benign tumors developed by c-Myc single transgenic mice. The molecular mechanisms underlying downregulation of E-cadherin in c-Myc tumors remain poorly understood at present. No $\mathrm{LOH}$ at the Ecadherin locus was detected in c-Myc HCCs, whereas only a subset of c-Myc tumors displayed hypermethylation of the E-cadherin promoter. Furthermore, no association was detected between E-cadherin downregulation and protein levels of transcriptional repressors Snail, Slug or the tumor suppressor WT1, in disagreement with the finding that overexpression of Snail suppresses E-cadherin in human HCC. ${ }^{15,18}$ In c-Myc HCCs, it is possible that E-cadherin loss might depend upon genetic mutations in the E-cadherin gene or by complex mechanisms, mainly operating at the transcriptional level. However, it is more likely that c-Myc overexpression directly downregulates E-cadherin. Although c-Myc activates expression of the Ecadherin gene in epithelial cells, ${ }^{48}$ only wild-type a

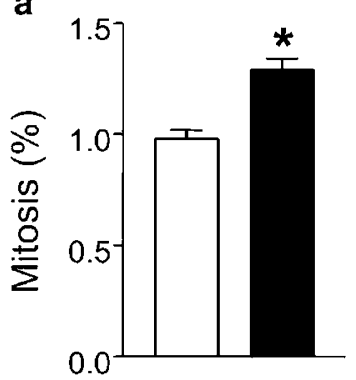

d

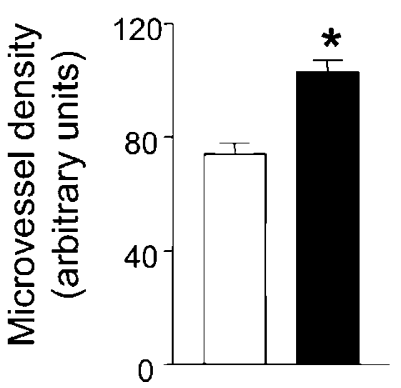

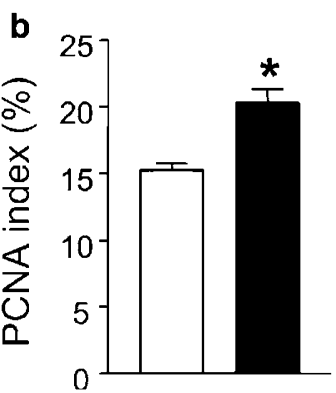

e

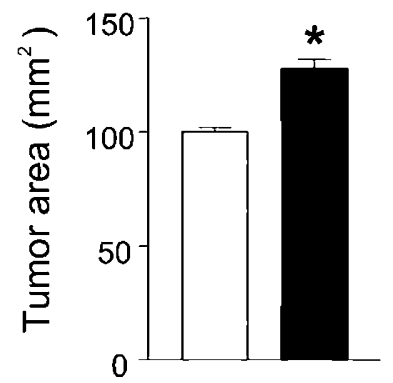

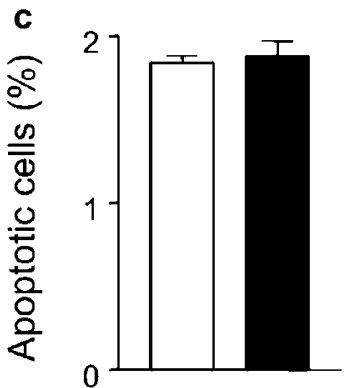

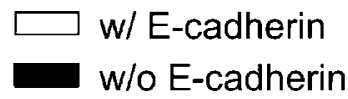

Figure 4 Effect of E-cadherin downregulation on tumor growth and vascularity in c-Myc HCCs. (a) mitotic index; (b) PCNA labeling index; (c) apoptotic index; (d) MVD; (e) tumor size. Each bar represents mean + s.e. $(n=8)$. 
c-Myc is able to transactivate the E-cadherin promoter, whereas the two isoforms of the c-Myc protein alone repress the same promoter. $^{49}$ Therefore, disruption of the ratio between the two c-Myc proteins induced by the c-Myc transgene might suppress E-cadherin expression in c-Myc malignant lesions. ${ }^{49}$ In accordance with this hypothesis, conditional induction of the c-Myc transgene led to suppression of E-cadherin in a pancreatic model of $\beta$-cell oncogenesis, suggesting that E-cadherin downregulation is a direct consequence of c-Myc activation. ${ }^{50}$ Only c-Myc and a minority of c-Myc/E2F1 HCCs displayed loss of E-cadherin, whereas c-Myc coexpression with TGF- $\alpha$ or E2F1 resulted more often in E-cadherin upregulation. Nonetheless, the increase of E-cadherin levels might also have a distinct functional role during tumor progression of the more malignant c-Myc/ TGF- $\alpha$, C-Myc/E2F1, and E2F1 HCCs. Indeed, Ecadherin expression seems to be beneficial for intraepithelial expansion and invasiveness in a variety of solid tumors, as well as for intrahepatic metastasis of HCC. ${ }^{19-22}$ Furthermore, previous studies showed that human HCC can be subdivided into two distinct categories which are characterized by loss or upregulation of E-cadherin, respectively. ${ }^{19,51}$ Importantly, the latter group was correlated significantly with the invasive potential of tumor cells, ${ }^{19}$ further supporting the theory that Ecadherin overexpression is required at some steps of the tumor process. Therefore, E-cadherin might play different and apparently opposite roles, which depend on specific tumor requirements in human and murine liver carcinogenesis. A number of studies have previously investigated the relationship among E-cadherin levels, cell proliferation and apoptosis in a variety of models, but not in mouse HCC. Here, we report for the first time that the loss of E-cadherin is associated with increased cell proliferation in c-Myc HCCs, whereas the apoptotic rate is not affected by E-cadherin downregulation. Although the molecular mechanisms underlying the promotion of cell proliferation in c-Myc HCCs remain to be addressed, we have identified HIF-1 $\alpha$ and VEGF as potential target genes of E-cadherin disruption. All HCCs from the four transgenic lines displayed upregulation of HIF-1 $\alpha$, but the highest expression levels were detected in c-Myc and cMyc/E2F1 tumors that had lost E-cadherin. These data suggest that downregulation of E-cadherin might depend upon HIF- $1 \alpha$ upregulation, as recently described in ovarian carcinoma cells. ${ }^{36}$ However, it is also possible that E-cadherin downregulates HIF- $1 \alpha$ and E-cadherin reduction leads to HIF- $1 \alpha$ upregulation. HIF-1 $\alpha$ is a heterodimeric transcription factor that regulates the transcription of genes involved in angiogenesis, glucose metabolism, cell differentiation and migration, cell proliferation and viability, $\mathrm{CO}_{2}$ metabolism, and ATP production. ${ }^{52}$ Since these functions are essential for tumor progression, HIF- $1 \alpha$ upregulation might represent a fundamental step during c-Myc hepatocarcinogenesis. In addition, c-Myc HCCs exhibited a remarkable induction of VEGF, in accordance with previous data showing that c-Myc is angiogenic in multiple cell types by its ability to induce VEGF. ${ }^{53}$ Again, VEGF upregulation was more pronounced in tumors with low levels of E-cadherin, suggesting that loss of E-cadherin facilitates VEGF upregulation. Strikingly, VEGF levels were very low in the more aggressive HCCs from E2F1, c-Myc/TGF- $\alpha$ and $\mathrm{c}-\mathrm{Myc} / \mathrm{E} 2 \mathrm{~F} 1$, suggesting that angiogenesis is promoted by other growth factors in these accelerated models of liver cancer. In accordance with this hypothesis, previous reports ${ }^{54,55}$ have shown that VEGF expression did not correlate with angiogenesis or any clinicopathological features of human HCC, suggesting that angiogenesis of HCC may be mediated by other proangiogenic molecules. Indeed, more recent findings indicate that expression of angiopoietin 2, tissue factor and angiogenin are associated with tumor vascularity in HCC. ${ }^{56-58}$ Similarly, our preliminary data showed that induction of different angiogenin isoforms correlated with the adenoma/carcinoma transition in c-Myc/TGF- $\alpha$ liver tumors. ${ }^{59}$

In addition to its adhesive properties, E-cadherin displays the potential to suppress cell proliferation and transformation by inhibiting $\mathrm{Wnt} / \beta$ catenin signaling. ${ }^{41-43}$ Therefore, downregulation of E-cadherin is a major mechanism underlying nuclear translocation of $\beta$-catenin. In accordance with human HCC, ${ }^{19,48}$ our present data show that $\beta$-catenin activation is not correlated with loss of E-cadherin, suggesting that other members of the cadherin family might retain $\beta$-catenin in membranous complexes. Importantly, activation of $\beta$-catenin and loss of E-cadherin represent two distinct molecular mechanisms involved in c-Myc hepatocarcinogenesis providing advantages at the early and late stages of tumor development respectively.

In summary, the present study shows that, similar to human HCC, E-cadherin levels identify two distinct categories of liver cancer in transgenic mice. The first group, which is mainly represented by c-Myc transgenic mice, displayed loss of E-cadherin during tumor progression. Downregulation of E-cadherin was associated with higher levels of HIF- $1 \alpha$ and VEGF proteins, increased cell proliferation, larger tumor size and more elevated MVD. These data suggest that loss of E-cadherin might favor the growth and invasion by inducing proliferation under hypoxic conditions and angiogenesis in the relatively benign c-Mycinduced hepatocarcinogenesis. In contrast, the second group, best exemplified by c-Myc/TGF- $\alpha$ transgenic mice, showed marked upregulation of E-cadherin starting from preneoplastic lesions. Whether E-cadherin upregulation also promotes growth and/or survival of neoplastic hepatocytes remains to be determined. 


\section{References}

1 Hussain SP, Harris CC. Molecular epidemiology and carcinogenesis: endogenous and exogenous carcinogens. Mutat Res 2000;462:311-322.

2 Shimada M, Takenaka K, Gion T, et al. Prognosis of recurrent hepatocellular carcinoma: a 10-year surgical experience in Japan. Gastroenterology 1996;111: $720-726$.

3 el-Assal ON, Yamanoi A, Soda Y, et al. Proposal of invasiveness score to predict recurrence and survival after curative hepatic resection for hepatocellular carcinoma. Surgery 1997;122:571-577.

4 Toyosaka A, Okamoto E, Mitsunobu M, et al. Intrahepatic metastases in hepatocellular carcinoma: evidence for spread via the portal vein as an efferent vessel. Am J Gastroenterol 1996;91:1610-1615.

5 Kemler R. From cadherins to catenins: cytoplasmic protein interactions and regulation of cell adhesion. Trends Genet 1993;9:317-321.

6 Behrens J, Frixen U, Schipper J, et al. Cell adhesion in invasion and metastasis. Semin Cell Biol 1992;3: 169-178.

7 Bracke ME, Van Roy FM, Mareel MM. The E-cadherin/ catenin complex in invasion and metastasis. Curr Top Microbiol Immunol 1996;213:123-161.

8 Christofori G, Semb H. The role of the cell-adhesion molecule E-cadherin as a tumour-suppressor gene. Trends Biochem Sci 1999;24:73-76.

9 Endo K, Ueda T, Ueyama J, et al. Immunoreactive E-cadherin, alpha-catenin, beta-catenin, and gamma-catenin proteins in hepatocellular carcinoma: relationships with tumor grade, clinicopathologic parameters, and patients' survival. Hum Pathol 2000; 31:558-565.

10 Takeichi M. Cadherins in cancer: implications for invasion and metastasis. Curr Opin Cell Biol 1993;5:806-811.

11 Chen WC, Obrink B. Cell-cell contacts mediated by E-cadherin (uvomorulin) restrict invasive behavior of L-cells. J Cell Biol 1991;114:319-327.

12 St Croix B, Sheehan C, Rak JW, et al. E-cadherindependent growth suppression is mediated by the cyclin-dependent kinase inhibitor p27 (KIP1). J Cell Biol 1998;142:557-571.

13 Perl AK, Wilgenbus P, Dahl U, et al. A causal role for E-cadherin in the transition from adenoma to carcinoma. Nature 1998;392:190-193.

14 Berx G, Becker KF, Hofler H, et al. Mutations of the human E-cadherin (CDH1) gene. Hum Mutat 1998; 12:226-237.

15 Jiao W, Miyazaki K, Kitajima Y. Inverse correlation between E-cadherin and Snail expression in hepatocellular carcinoma cell lines in vitro and in vivo. Br J Cancer 2002;86:98-101.

16 Kanai Y, Ushijima S, Hui AM, et al. The E-cadherin gene is silenced by CpG methylation in human hepatocellular carcinomas. Int J Cancer 1997;71: 355-359.

17 Matsumura T, Makino R, Mitamura K. Frequent downregulation of E-cadherin by genetic and epigenetic changes in the malignant progression of hepatocellular carcinomas. Clin Cancer Res 2001;7:594-599.

18 Sugimachi K, Tanaka S, Kameyama T, et al. Transcriptional repressor snail and progression of human hepatocellular carcinoma. Clin Cancer Res 2003;9: 2657-2664.
19 Wei Y, Van Nhieu JT, Prigent S, et al. Altered expression of E-cadherin in hepatocellular carcinoma: correlations with genetic alterations, beta-catenin expression, and clinical features. Hepatology 2002;36: 692-701

20 Bignell GR, Warren W, Seal S, et al. Identification of the familial cylindromatosis tumour-suppressor gene. Nat Genet 2000;25:160-165.

21 Osada T, Sakamoto M, Ino Y, et al. E-cadherin is involved in the intrahepatic metastasis of hepatocellular carcinoma. Hepatology 1996;24:1460-1467.

22 Tomlinson JS, Alpaugh ML, Barsky SH. An intact overexpressed E-cadherin/alpha,beta-catenin axis characterizes the lymphovascular emboli of inflammatory breast carcinoma. Cancer Res 2001;61:5231-5241.

23 Murakami H, Sanderson ND, Nagy P, et al. Transgenic mouse model for synergistic effects of nuclear oncogenes and growth factors in tumorigenesis: interaction of c-myc and transforming growth factor- $\alpha$ in hepatic oncogenesis. Cancer Res 1993;53:1719-1723.

24 Conner EA, Lemmer ER, Omori M, et al. Dual functions of E2F-1 in a transgenic mouse model of liver carcinogenesis. Oncogene 2000;19: 5054-5062.

25 Conner EA, Lemmer ER, Sanchez A, et al. E2F1 blocks and c-Myc accelerates hepatic ploidy in transgenic mouse models. Biochem Biophys Res Commun 2003; 302:114-120.

26 Frith CH, Ward JM, Turusov VS. Tumours of the liver. In: Turusov V, Mohr U (eds). Pathology of Tumours in Laboratory Animals, Vol. 2, IARC Scientific Publications: Lyon, France, 1994, pp 223-270.

27 Laird PW, Zijderveld A, Linders K, et al. Simplified mammalian DNA isolation procedure. Nucleic Acids Res 1991;19:4293.

28 Calvisi DF, Factor VM, Loi R, et al. Activation of $\beta$ catenin during hepatocarcinogenesis in transgenic mouse models: relationship to phenotype and tumor grade. Cancer Res 2001;61:2085-2091.

29 Faraldo ML, Rodrigo I, Behrens J, et al. Analysis of the E-cadherin and P-cadherin promoters in murine keratinocyte cell lines from different stages of mouse skin carcinogenesis. Mol Carcinogon 1997;20:33-47.

30 Rodrigo I, Cato AC, Cano A. Regulation of E-cadherin gene expression during tumor progression: the role of a new Ets-binding site and the E-pal element. Exp Cell Res 2000;248:358-371.

31 Thorgeirsson SS, Grisham JW. Molecular pathogenesis of human hepatocellular carcinoma. Nat Genet 2002;31:339-346.

32 Batlle E, Sancho E, Franci C, et al. The transcription factor snail is a repressor of E-cadherin gene expression in epithelial tumour cells. Nat Cell Biol 2000;2:84-89.

33 Comijn J, Berx G, Vermassen P, et al. The two-handed $\mathrm{E}$ box binding zinc finger protein SIP1 downregulates E-cadherin and induces invasion. Mol Cell 2001;7: 1267-1278.

34 Hajra KM, Chen DY, Fearon ER. The SLUG zinc-finger protein represses E-cadherin in breast cancer. Cancer Res 2002;62:1613-1618.

35 Perez-Moreno MA, Locascio A, Rodrigo I, et al. A new role for E12/E47 in the repression of E-cadherin expression and epithelial-mesenchymal transitions. J Biol Chem 2001;276:27424-27431.

36 Imai T, Horiuchi A, Wang C, et al. Hypoxia attenuates the expression of E-cadherin via up-regulation of 
SNAIL in ovarian carcinoma cells. Am J Pathol 2003;163:1437-1447.

37 Hosono S, Gross I, English MA, et al. E-cadherin is a WT1 target gene. J Biol Chem 2000;275:10943-10953.

38 Forsythe JA, Jiang $\mathrm{BH}$, Iyer $\mathrm{NV}$, et al. Activation of vascular endothelial growth factor gene transcription by hypoxia-inducible factor 1. Mol Cell Biol 1996; 16:4604-4613.

39 Shweiki D, Itin A, Soffer D, et al. Vascular endothelial growth factor induced by hypoxia may mediate hypoxia-initiated angiogenesis. Nature 1992;359: 843-845.

40 Calvisi DF, Factor VM, Ladu S, et al. Disruption of $\beta$ catenin pathway or genomic instability define two distinct categories of liver cancer in transgenic mice. Gastroenterology 2004;126:1374-1386.

41 Baudry D, Cabanis MO, Patte C, et al. Cadherins in Wilms' tumor: E-cadherin expression despite absence of WT1. Anticancer Res 2003;23:475-478.

42 Gottardi CJ, Wong E, Gumbiner BM. E-cadherin suppresses cellular transformation by inhibiting betacatenin signaling in an adhesion-independent manner. J Cell Biol 2001;153:1049-1060.

43 Orsulic S, Huber O, Aberle H, et al. E-cadherin binding prevents beta-catenin nuclear localization and betacatenin/LEF-1-mediated transactivation. J Cell Sci 1999; 112:1237-1245.

44 De La Coste A, Romagnolo B, Billuart P, et al. Somatic mutations of the beta-catenin gene are frequent in mouse and human hepatocellular carcinomas. Proc Natl Acad Sci USA 1998;95:8847-8851.

45 Renard CA, Fourel G, Bralet MP, et al. Hepatocellular carcinoma in WHV/N-myc2 transgenic mice: oncogenic mutations of beta-catenin and synergistic effect of p53 null alleles. Oncogene 2000;19:2678-2686.

46 Sadot E, Simcha I, Shtutman M, et al. Inhibition of beta-catenin-mediated transactivation by cadherin derivatives. Proc Natl Acad Sci USA 1998;95: 15339-15344.

47 Conacci-Sorrell M, Zhurinsky J, Ben-Ze'ev A. The cadherin-catenin adhesion system in signaling and cancer. J Clin Invest 2002;109:987-991.

48 Batsche E, Muchardt C, Behrens J, et al. RB and c-Myc activate expression of the E-cadherin gene in epithelial cells through interaction with transcription factor AP-2. Mol Cell Biol 1998;18:3647-3658.
49 Batsche E, Cremisi C. Opposite transcriptional activity between the wild type c-myc gene coding for c-Myc1 and c-Myc2 proteins and c-Myc1 and c-Myc2 separately. Oncogene 2000;18:5662-5671.

50 Pelengaris S, Khan M, Evan GI. Suppression of Mycinduced apoptosis in beta cells exposes multiple oncogenic properties of Myc and triggers carcinogenic progression. Cell 2001;109:321-334.

51 Ihara A, Koizumi H, Hashizume R, et al. Expression of epithelial cadherin and alpha- and beta-catenins in nontumoral livers and hepatocellular carcinomas. Hepatology 1996;23:1441-1447.

52 Semenza GL. HIF-1 and tumor progression: pathophysiology and therapeutics. Trends Mol Med 2002;8: S62-S67.

53 Baudino TA, McKay C, Pendeville-Samain H, et al. cMyc is essential for vasculogenesis and angiogenesis during development and tumor progression. Genes Dev 2002;16:2530-2543.

54 el-Assal ON, Yamanoi A, Soda Y, et al. Clinical significance of microvessel density and vascular endothelial growth factor expression in hepatocellular carcinoma and surrounding liver: possible involvement of vascular endothelial growth factor in the angiogenesis of cirrhotic liver. Hepatology 1998;27: 1554-1562.

55 Yamaguchi R, Yano H, Nakashima Y, et al. Expression and localization of vascular endothelial growth factor receptors in human hepatocellular carcinoma and nonHCC tissues. Oncol Rep 2000;4:725-729.

56 Mitsuhashi N, Shimizu H, Ohtsuka M, et al. Angiopoietins and Tie-2 expression in angiogenesis and proliferation of human hepatocellular carcinoma. Hepatology 2003;37:1105-1113.

57 Poon RT, Lau CP, Ho JW, et al. Tissue factor expression correlates with tumor angiogenesis and invasiveness in human hepatocellular carcinoma. Clin Cancer Res 2003;9:5339-5345.

58 Hisai H, Kato J, Kobune M, et al. Increased expression of angiogenin in hepatocellular carcinoma in correlation with tumor vascularity. Clin Cancer Res 2003; 9:4852-4859.

59 Shiomi H, Lee JS, Hironaka K, et al. Differential gene expression in hepatocellular adenoma and carcinoma in c-Myc/TGF- $\alpha$ transgenic mice. Proc Am Assoc Cancer Res 2002;43:861. 